\title{
Mortality after transcatheter versus surgical aortic valve replacement: an updated meta-analysis of randomised trials
}

\author{
H. Takagi D for ALICE (All-Literature Investigation of Cardiovascular Evidence) Group - Y. Hari for ALICE (All- \\ Literature Investigation of Cardiovascular Evidence) Group - K. Nakashima for ALICE (All-Literature Investigation \\ of Cardiovascular Evidence) Group - T. Kuno for ALICE (All-Literature Investigation of Cardiovascular Evidence) \\ Group · T. Ando for ALICE (All-Literature Investigation of Cardiovascular Evidence) Group
}

Published online: 12 March 2020

(C) The Author(s) 2020

\begin{abstract}
Background To determine whether transcatheter aortic valve implantation (TAVI) improves early (30-day) and midterm (1-year) mortality compared with surgical aortic valve replacement (SAVR), we performed an updated meta-analysis of all the currently available randomised controlled trials (RCTs).

Methods To identify all RCTs providing both 30-day and 1-year mortality after TAVI versus SAVR, PubMed and ClinicalTrials.gov were searched up to and including July 2019. A risk difference (RD) and its 95\% confidence interval were generated using data of prespecified outcomes in both the TAVI and SAVR groups. Study-specific estimates were pooled using inverse variance-weighted averages of $\mathrm{RDs}$ in the random-effects model.

Results We identified seven eligible high-quality RCTs including a total of 7631 as-treated patients. Pooled analyses demonstrated significantly lower 30-day (RD $-0.60 \% ; p=0.046)$ and 1 -year all-cause mortality (RD
\end{abstract}

Electronic supplementary material The online version of this article (https://doi.org/10.1007/s12471-020-01378-1) contains supplementary material, which is available to authorized users.

H. Takagi $(\bowtie) \cdot$ Y. Hari $\cdot$ K. Nakashima

Department of Cardiovascular Surgery, Shizuoka Medical Center, Shizuoka, Japan

Department of Cardiovascular Surgery, Kitasato University School of Medicine, Sagamihara, Japan

kfgth973@ybb.ne.jp

\section{T. Kuno}

Department of Medicine, Mount Sinai Beth Israel Medical Center, New York, NY, USA

\section{T. Ando}

Division of Interventional Cardiology, Department of Cardiology, New York Presbyterian Hospital/Columbia University Medical Center, New York, NY, USA
$-1.12 \% ; p=0.03)$ after TAVI than after SAVR. No funnel plot asymmetry was detected for 30-day and 1-year mortality. Meta-regression analyses indicated that RDs of 30-day and 1-year mortality between TAVI and SAVR were not modulated by mean Society of Thoracic Surgeons Predicted Risk of Mortality score. Bleeding complications at 30 days and 1 year and stage $2 / 3$ acute kidney injury at 30 days were significantly less frequent after TAVI than after SAVR, whereas major vascular complications and new permanent pacemaker implantation at 30 days and 1 year were significantly more frequent after TAVI than after SAVR.

Conclusion The best evidence from the present metaanalysis of all the currently available RCTs suggests that TAVI may reduce 30-day and 1-year all-cause mortality compared with SAVR.

\section{What's new?}

- To determine whether transcatheter aortic valve implantation (TAVI) improves early (30-day) and midterm (1-year) mortality compared with surgical aortic valve replacement (SAVR), we performed an updated meta-analysis of all the currently available randomised controlled trials (RCTs).

- We identified seven eligible high-quality RCTs including a total of 7631 as-treated patients.

- None of the included RCTs showed significantly lower all-cause mortality after TAVI than after SAVR.

- Pooled analyses demonstrated significantly lower 30-day [risk difference (RD) $-0.60 \%$; $p=0.046$ ] and 1-year all-cause mortality (RD $-1.12 \%$; $p=0.03$ ) after TAVI than after SAVR. 
Keywords Meta-analysis - Randomised controlled trial - Surgical aortic valve replacement .

Transcatheter aortic valve implantation

\section{Introduction}

Because it is a less invasive procedure, transcatheter aortic valve implantation (TAVI) was introduced as a substitute for surgical aortic valve replacement (SAVR) in high surgical risk patients with severe aortic stenosis (AS) and was expected to achieve at least equivalent or if possible better postprocedural prognosis. As far as we know, however, neither randomised controlled trials (RCTs) of TAVI versus SAVR nor meta-analyses [1-4] of RCTs have reported significantly lower mortality after TAVI than after SAVR to date. Recently (in 2019), two novel RCTs, the Evolut Low Risk trial [5] and the Placement of Aortic Transcatheter Valves (PARTNER) 3 trial [6], provided outcomes after TAVI versus SAVR. In the present article, to determine whether TAVI improves early (30day) and midterm (1-year) mortality compared with SAVR, we performed an updated meta-analysis of all the currently available RCTs including the two abovementioned recently reported RCTs $[5,6]$.

\section{Methods}

To identify all RCTs providing both 30-day and 1-year mortality after TAVI versus SAVR for AS patients, PubMed (https://www.ncbi.nlm.nih.gov/pubmed/) and ClinicalTrials.gov (https://clinicaltrials.gov/ct2/ home) were searched up to and including July 2019. Search terms included 'transcatheter', 'aortic valve' 'implantation(s) or replacement(s)' and 'randomised'. Studies meeting the following criteria were included in a meta-analysis: the design was an RCT; the study population consisted of AS patients; patients were randomised to TAVI versus SAVR; outcomes included both 30-day and 1-year all-cause mortality. A risk difference (RD) and its 95\% confidence interval (CI) were generated using data of prespecified outcomes in both the TAVI and SAVR groups. Study-specific estimates were pooled using inverse variance-weighted averages of RDs in the random-effects model. In the present study, the primary end point was all-cause mortality, and the secondary end points included myocardial infarction, stroke, bleeding complications, acute kidney injury (AKI), vascular complications, and new permanent pacemaker implantation (PMI). When the number of studies reporting an end point was $<3$, we did not perform pooled analysis for the end point. Funnel plot asymmetry (suggesting publication bias) was mathematically examined using the linear regression test. To assess whether mean surgical risk [Society of Thoracic Surgeons Predicted Risk of Mortality (STS-PROM) score] of patients and proportion of patients undergoing trans(ilio)femoral TAVI (TF-TAVI) modulate study-specific estimates (RDs of mortality between TAVI and SAVR), a random-effects restricted-maximum likelihood meta-regression analysis was conducted. All analyses were performed using Review Manager version 5.3 (available from http://tech.cochrane.org/revman) and Comprehensive Meta-Analysis version 3 (Biostat, Englewood, NJ, USA).

\section{Results}

The STACCATO trial (prospective, randomised trial of transapical transcatheter aortic valve implantation vs surgical aortic valve replacement in operable elderly patients with aortic stenosis) [7] was not registered in ClinicalTrials.gov. Furthermore, the study was unexpectedly terminated after including only 70 patients and did not report 1-year outcomes. Thus, we decided to exclude this truncated RCT [7], and accordingly seven eligible high-quality RCTs ([5, 6, 8-12]; Tab. 1) were included in the present meta-analysis. Three RCTs (Evolut Low Risk [5], Nordic Aortic Valve Intervention (NOTION) [8], and PARTNER 3 [6]) consisted of patients at low surgical risk (STS-PROM $<4 \%$ ), three RCTs (PARTNER 2 [10], Surgical Replacement and Transcatheter Aortic Valve Implantation (SURTAVI) [11], and U.S. CoreValve [12]) were composed of those at intermediate surgical risk (STS-ROM 4-8\%), and only one RCT (PARTNER 1 [9]) was made up of those at high surgical risk (STS-ROM $\geq 8 \%$ ). The primary analysis in each RCT was conducted in the astreated population in five studies $[5,6,8,11,12]$ and in the intention-to-treat population in two studies [9, 10]. Hence, we determined to extract data in the astreated population from all the seven RCTs including a total of 7631 patients. The principal analysis of the present study pooled data from the as-treated population, and the sensitivity analysis combined data from the intention-to-treat population. We performed another sensitivity analysis excluding the PARTNER 1 trial [9] (including patients at high surgical risk) from the principal analysis (as-treated population) of the primary end point (all-cause mortality). Details of the primary and secondary end points are listed in Tab. 1 and Table S1 (Electronic Supplementary Material). Results of the principal and sensitivity analysis are summarised in Tab. 2.

None of the included RCTs showed significantly lower all-cause mortality after TAVI than after SAVR (Fig. 1). The principal analysis of the primary end point demonstrated significantly lower 30-day [RD $-0.60 \%$; $95 \%$ CI $-1.20 \%$ to $-0.01 \%$; $p=0.05(0.046$, calculated using Comprehensive Meta-Analysis version 3); $I^{2} 0 \%$ ] and 1-year all-cause mortality (RD $-1.12 \%$; $95 \%$ CI $-2.12 \%$ to $-0.11 \% ; p=0.03 ; l^{2} 0 \%$ ) after TAVI than after SAVR (Fig. 1). No funnel plot asymmetry was detected for 30-day ( $p=0.29$; Fig. 2 ) and 1-year mortality ( $p=0.26$; Fig. 3 ), which suggested no publication bias. Meta-regression analyses indicated that RDs of 30-day and 1-year mortality ( $p=0.73$; Fig. 5) 


\section{Original Article}

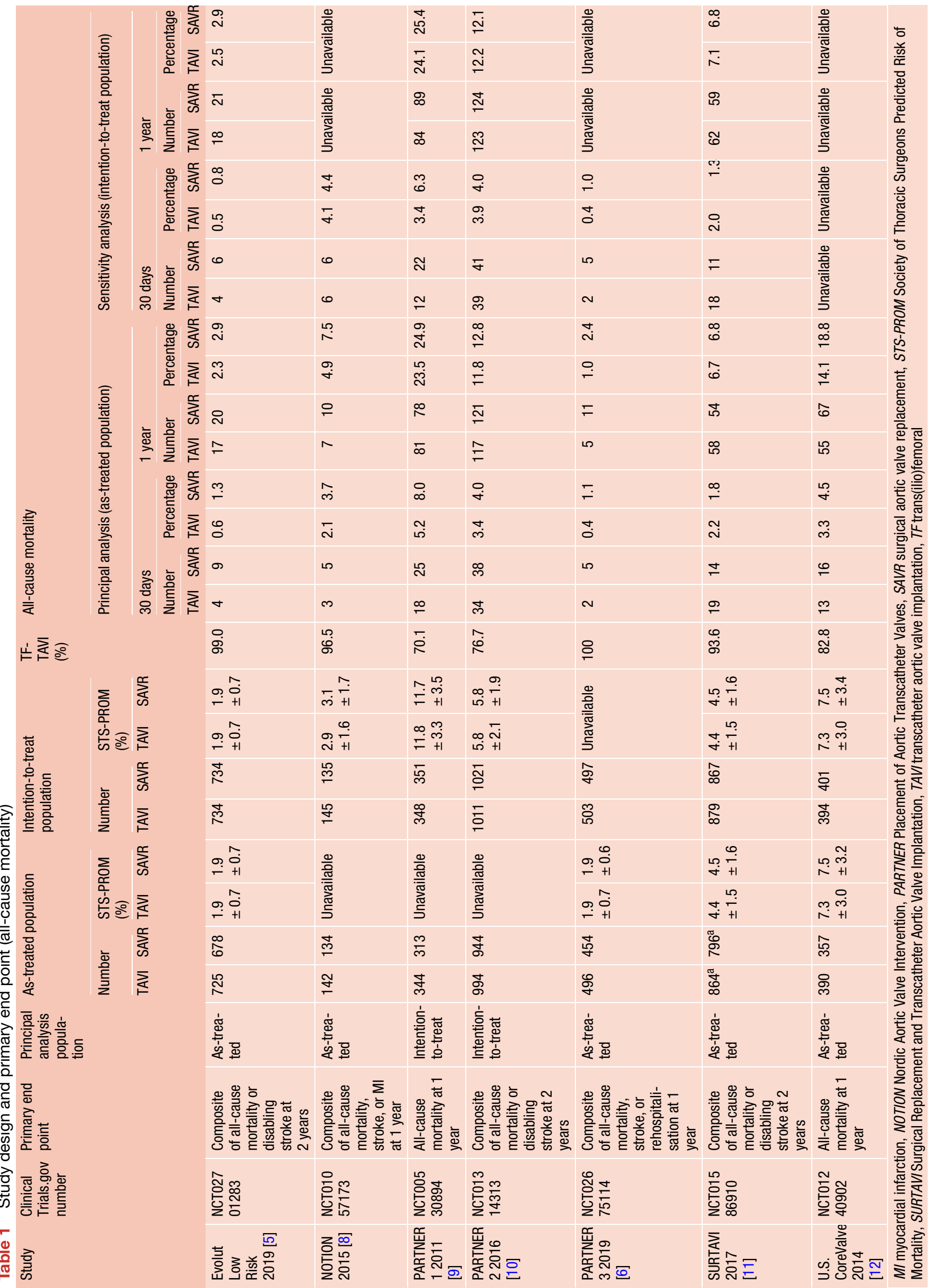




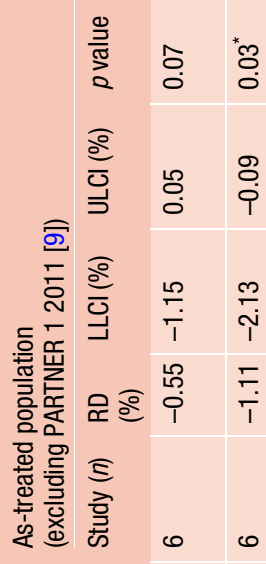

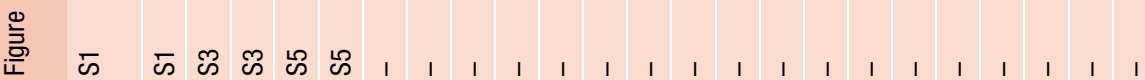

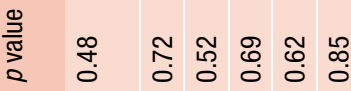

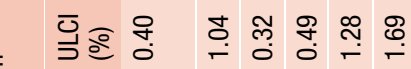

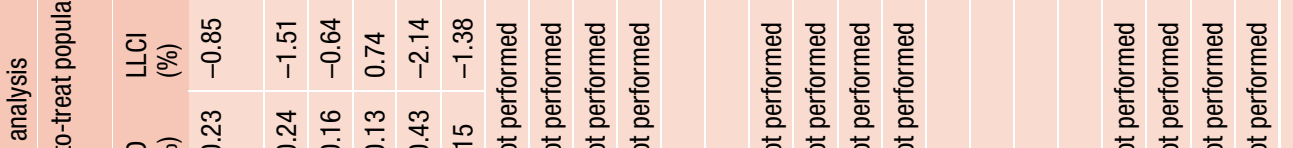

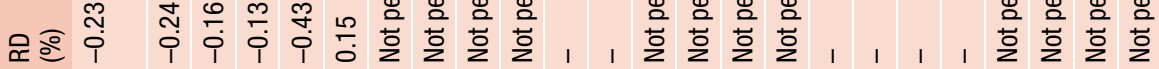

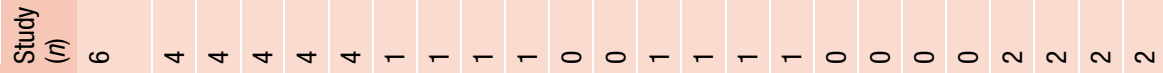

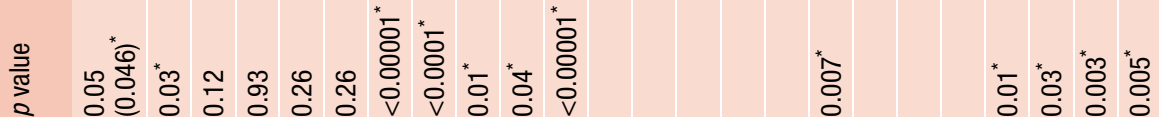

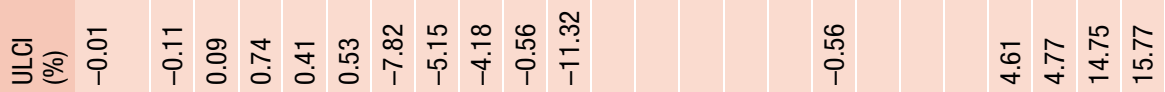
흥

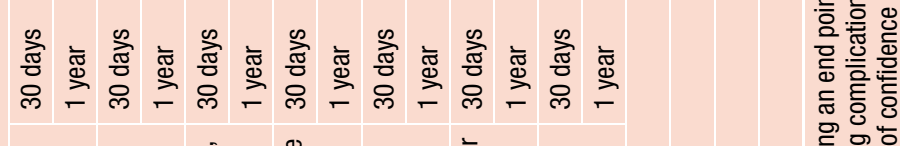

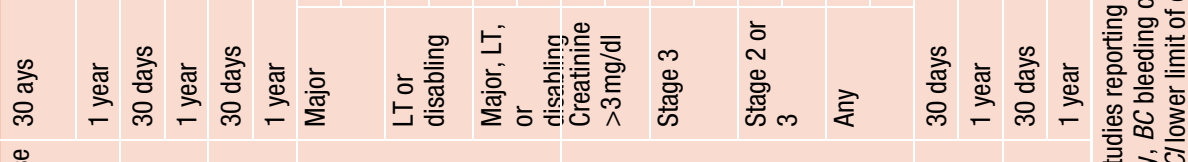

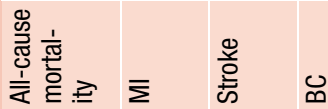
$\gtreqless \quad \sum_{i=1}^{\infty}$

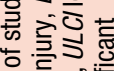

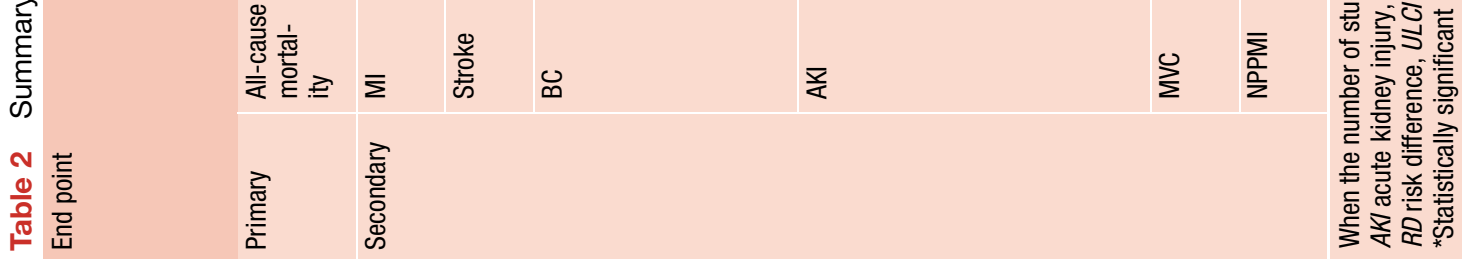


Fig. 1 Forest plot of the principal analysis (astreated population) of the primary end point: risk differences in 30-day and 1-year all-cause mortality between transcatheter aortic valve implantation (TAVI) and surgical aortic valve replacement $(S A V R)$. Cl confidence interval, $I V$ inverse variance, NOTION Nordic Aortic Valve Intervention, PARTNER Placement of Aortic Transcatheter Valves, SURTAVI Surgical Replacement and Transcatheter Aortic Valve Implantation

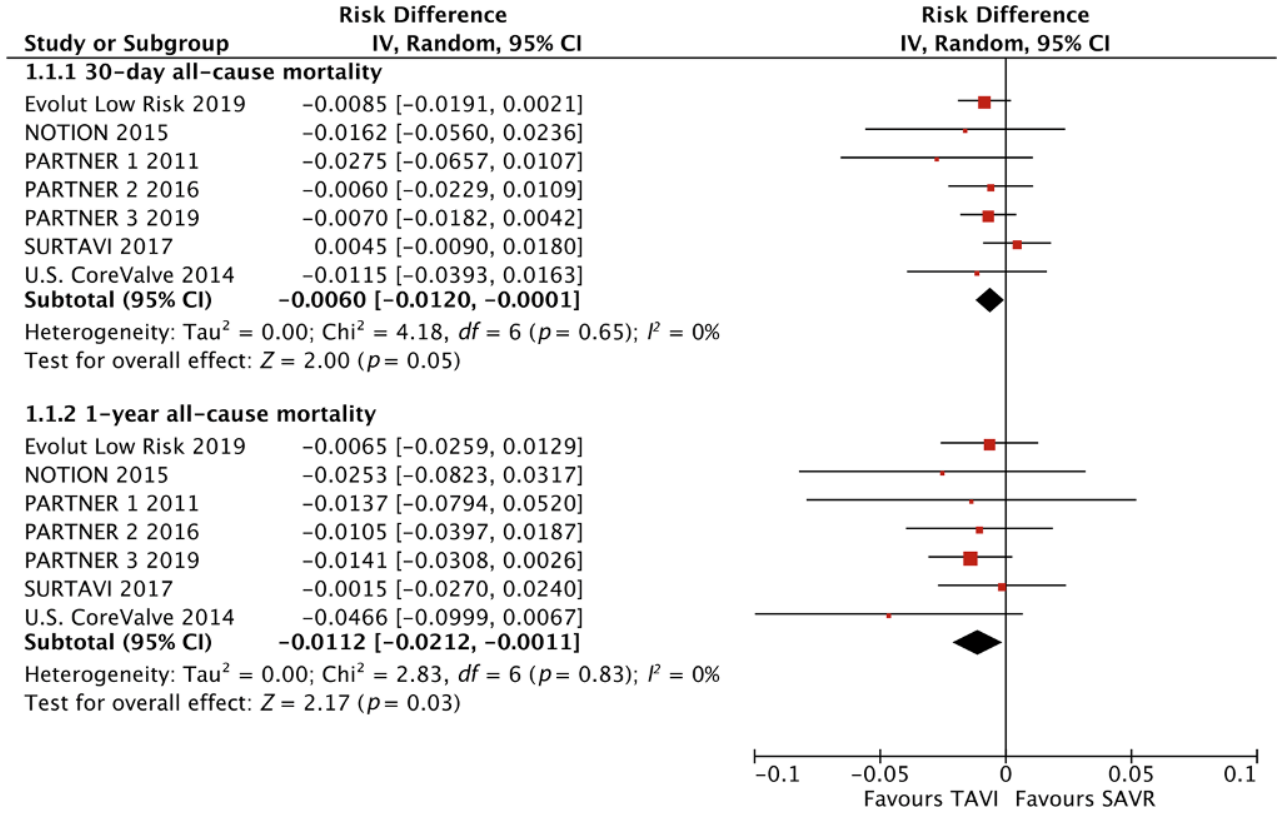

between TAVI and SAVR were not modulated by mean STS-PROM ( $p$ for 30-day/1-year mortality=0.82/0.73; Figs. 4 and 5) and proportion of patients undergoing TF-TAVI ( $p$ for 30-day/1-year mortality $=0.73 / 0.50$ ).

Results of the sensitivity analysis of the primary end point (all-cause mortality) are illustrated in Supplementary Fig. S1, and those of the principal and sensitivity analysis of the secondary end points (myocardial infarction, stroke, bleeding complications, AKI, vascular complications, and new permanent PMI) are diagramed in Supplementary Figs. S2-S10. Bleeding complications at 30 days and 1 year (Supplementary Figs. S6 and S7) and stage 2 or 3 AKI at 30 days (Supplementary Fig. S8) were significantly less frequent after TAVI than after SAVR, whereas major vascular complications (Supplementary Fig. S9) and new permanent PMI (Supplementary Fig. S10) at 30 days and 1 year were significantly more frequent after TAVI than after SAVR. There were no statistically significant differences in myocardial infarction (Supplementary Figs. S2 and S3) and stroke (Supplementary Figs. S4 and S5) at 30 days and 1 year between TAVI and SAVR.

\section{Discussion}

The present study is the first meta-analysis (of RCTs) demonstrating that TAVI improves 30-day and 1-year all-cause mortality compared with SAVR for AS patients. The absolute risk reduction was low, $0.60 \%$ for 30 -day mortality and $1.12 \%$ for 1 -year mortality, but statistically significant. The present findings must be novel because none of the included RCTs showed significantly lower all-cause mortality after TAVI than after SAVR.

In the present principal analysis, data in the astreated (not intention-to-treat) population were abstracted from each study and then combined because five of the seven RCTs principally analysed the astreated population. To draw an unbiased estimate of the effect of the randomised treatment on the outcome, in general, the intention-to-treat analysis is recommended [13]. If some participants do not receive the randomised treatment, however, the intention-totreat analysis may provide a biased estimate of the effect of the received treatment on the outcome. The as-treated analysis compares patients according to the received treatment rather than the randomised treatment, i.e. those who received the experimental treatment (whether or not they had been randomised to the experimental treatment) versus those who received the control treatment (whether or not they had been randomised to the control treatment) [13]. Thus, the as-treated analysis draws an unbiased estimate of the effect of the received treatment on the outcome. Clinicians or patients may be interested in whether the patient's prognosis improves if the patient receives the experimental treatment (not if the patient is randomised to the experimental treatment) [13].

We extracted RDs of mortality from each study and then combined them in the present meta-analysis. Although simplicity for interpretation purposes is recognised to be a qualitative property, an RD would be agreed to be a simple measure and thus easily understood [14]. An RD advantage of, for example, 10\% in the mortality rates of the experimental group relative to the control group is exactly equal to an $\mathrm{RD}$ disadvantage of $10 \%$ of the control group relative to the experimental group, which provides a symmetrical measure unaffected by labelling of study groups. In contradistinction to a risk or odds ratio estimate, an unbiased RD estimate is able to be gained from sample data based on the difference of two independent binomial variables [14]. 
Fig. 2 Funnel plot of the principal analysis (astreated population) of the primary end point: precision all-cause mortality between transcatheter aortic valve implantation and surgical aortic valve replacement by risk differences in 30-day

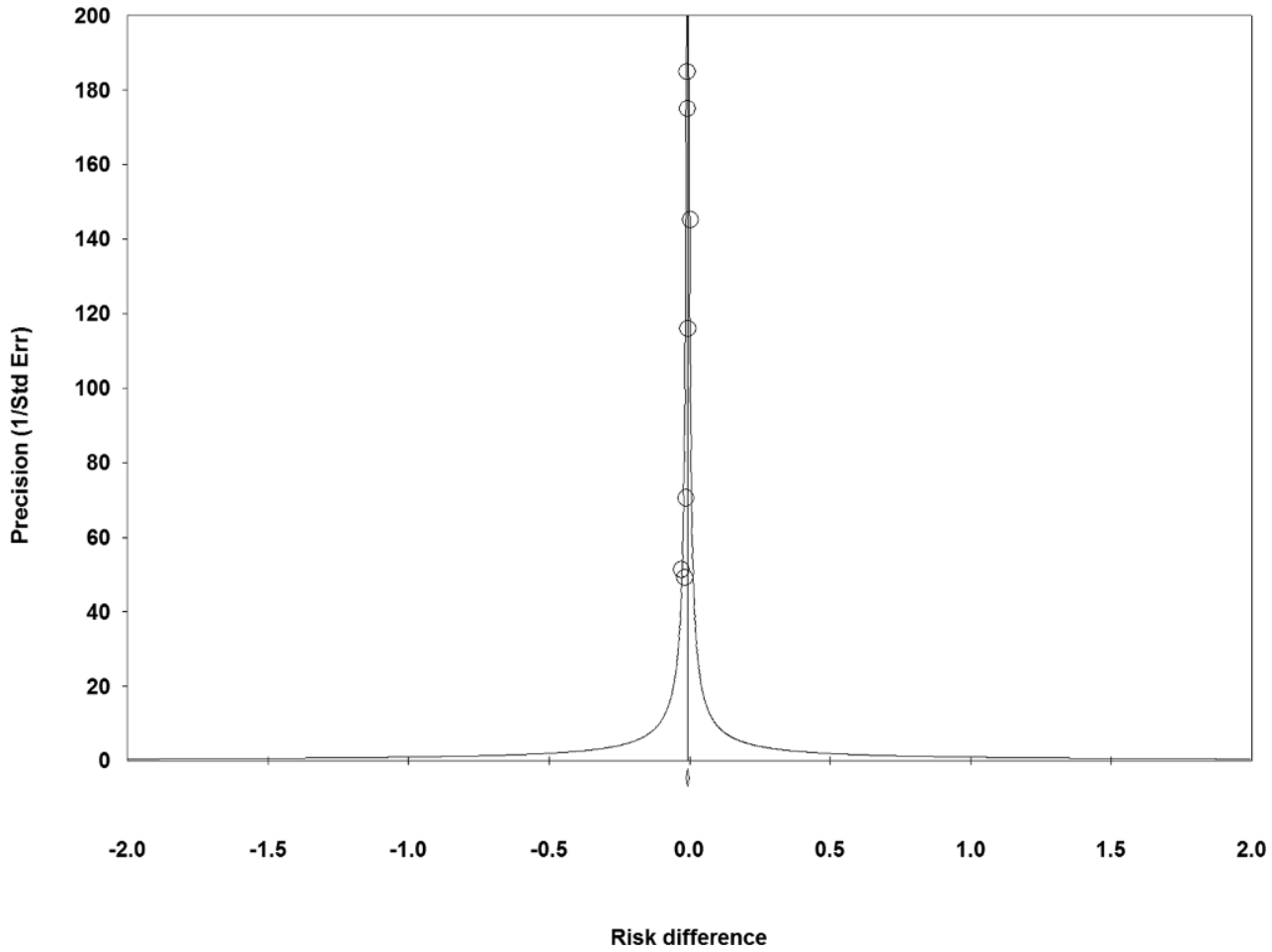

Fig. 3 Funnel plot of the principal analysis (astreated population) of the primary end point: precision by risk differences in 1-year all-cause mortality between transcatheter aortic valve implantation and surgical aortic valve replacement

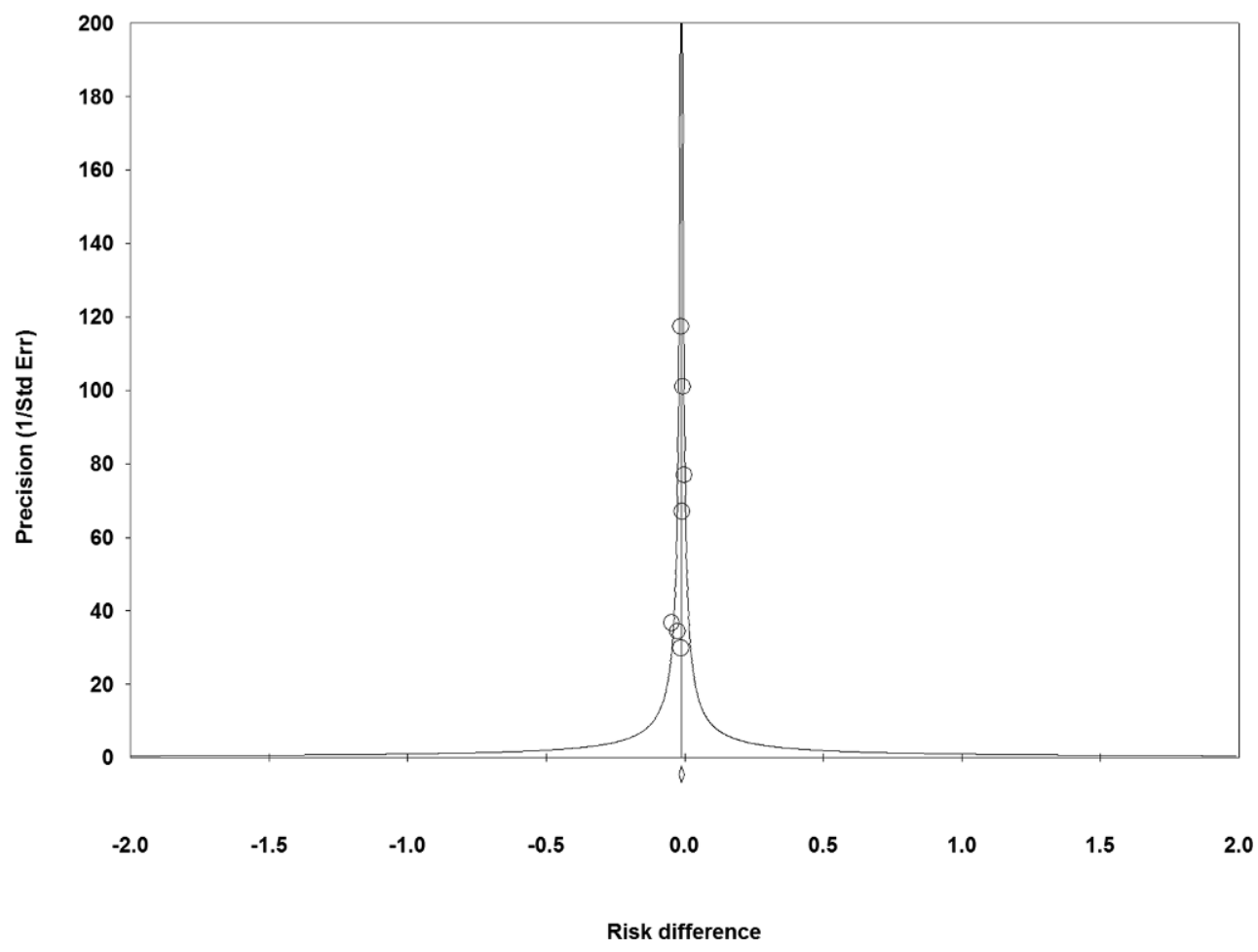

Significantly lower 30-day and 1-year all-cause mortality (Fig. 1) after TAVI than after SAVR (in the present principal analysis of the primary end point) could be explained by significantly less frequent bleeding complications at 30 days and 1 year (Supplementary Figs. S6 and S7) and stage 2 or 3 AKI (Supplementary Fig. S8) after TAVI than after SAVR (in the present principal analysis of the secondary end points). In the meta-analysis by Wang et al. [15] of 10 studies with a total of 3602 patients undergoing TAVI, bleeding complications were associated with a $323 \%$ increase in 30-day all-cause mortality [odds ratio (OR) 4.23; 95\% CI 2.80-6.40; $p<0.0001$ ], and major or life-threatening bleeding complications showed a $410 \%$ increase in 30-day all-cause mortality (OR 5.10; 95\% CI 3.17-8.19; $p<0.0001$ ). Furthermore, Liao 
Fig. 4 Meta-regression plot (meta-regression line with $95 \%$ confidence interval curves) of the principal analysis (as-treated population) of the primary end point: risk differences in 30-day all-cause mortality (between transcatheter aortic valve implantation and surgical aortic valve replacement) on Society of Thoracic Surgeons Predicted Risk of Mortality (STS-PROM) score

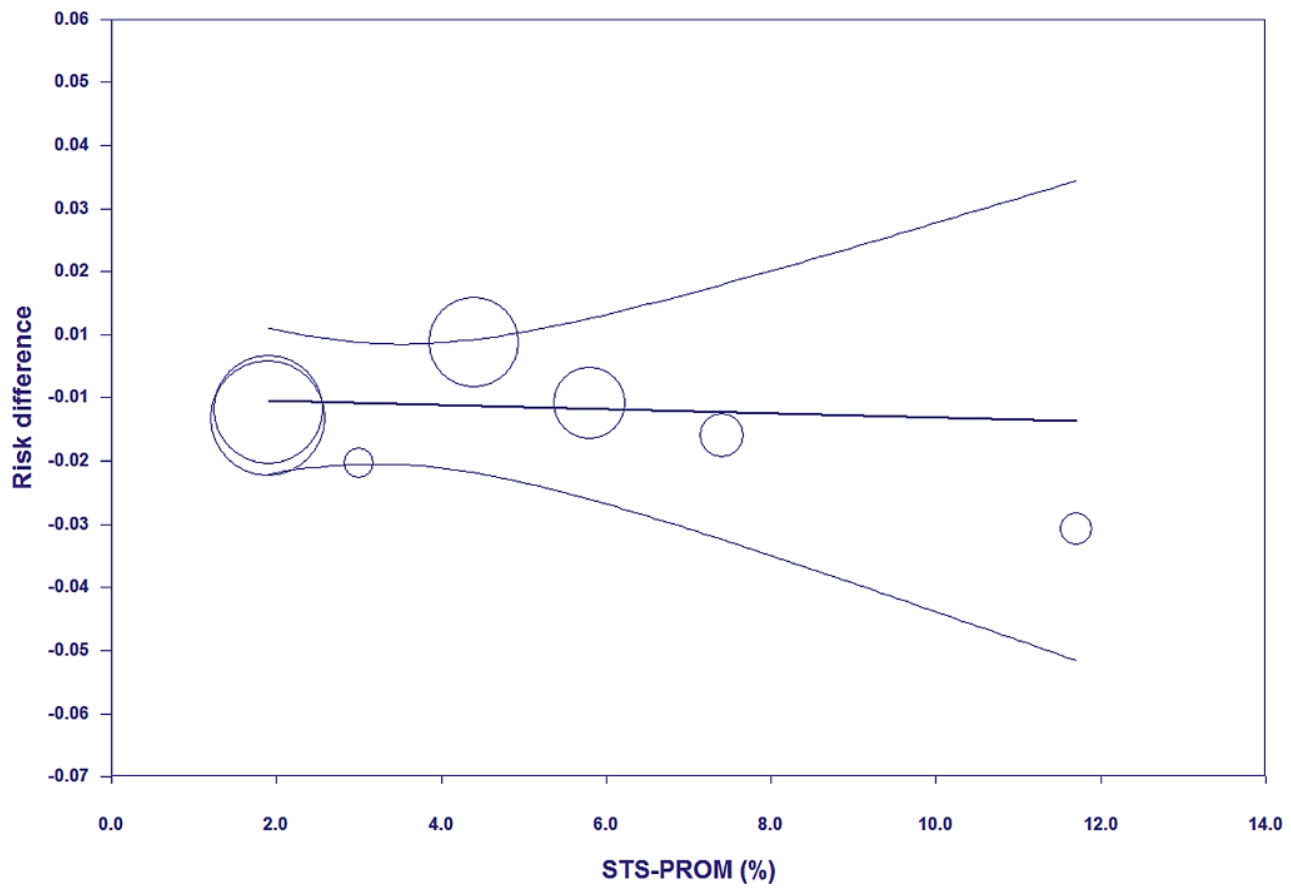

Fig. 5 Meta-regression plot (meta-regression line with $95 \%$ confidence interval curves) of the principal analysis (as-treated population) of the primary end point: risk differences in 1-year all-cause mortality (between transcatheter aortic valve implantation and surgical aortic valve replacement) on Society of Thoracic Surgeons Predicted Risk of Mortality (STS-PROM) score

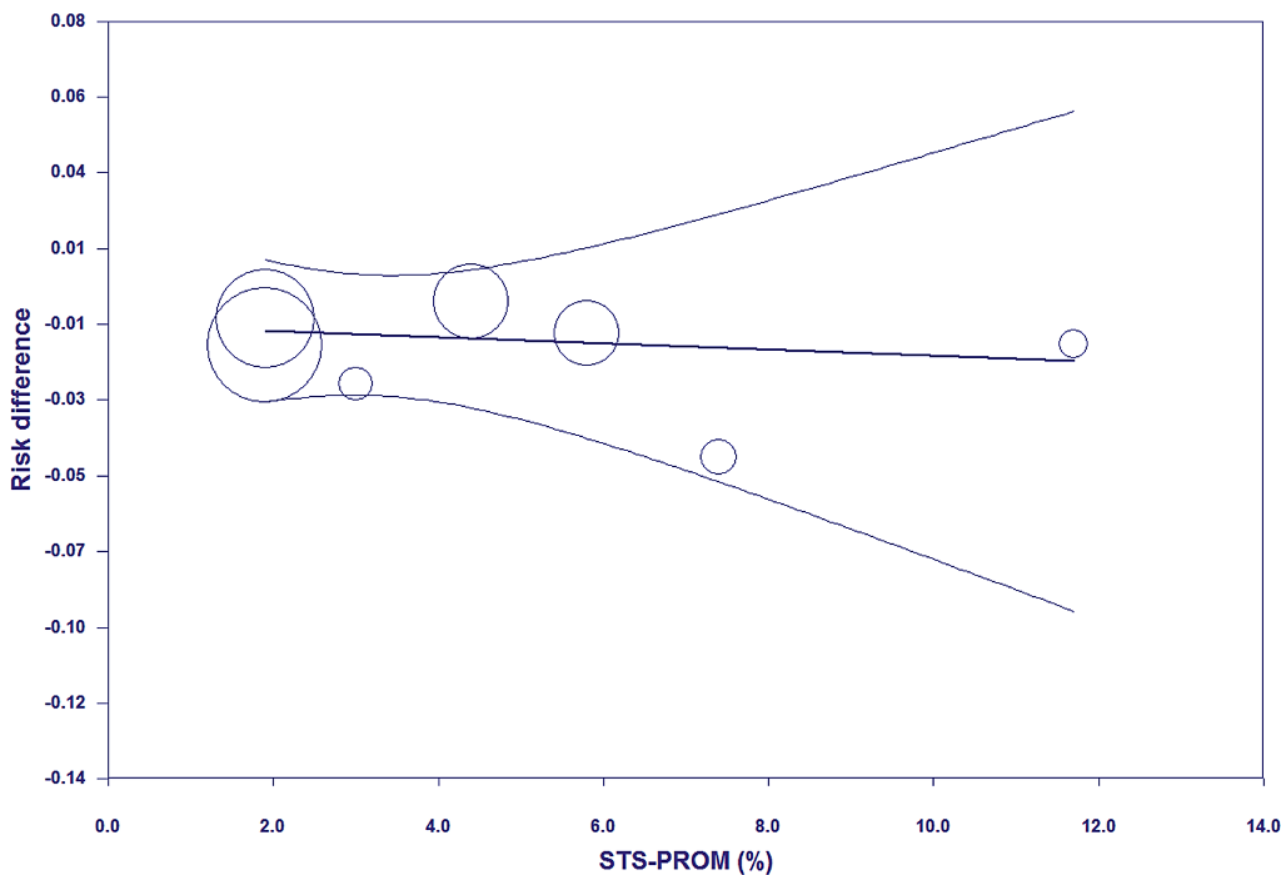

et al. [16] demonstrated, in their meta-analysis of 35 studies with a total of 13,256 patients undergoing TAVI, that the aggravating severity of AKI was progressively associated with short-term all-cause mortality (univariate OR of 30-day mortality for stage 1, 3.41; for stage 2, 4.0; for stage 3, 11.02; univariate OR of 1-year mortality for stage $1,1.95$; stage $2,2.82$; stage $3,7.34$ ). Even after controlling confounders, AKI was independently associated with a higher risk of both 30-day [multivariate hazard ratio (HR) 2.12; 95\% CI 1.59-2.83] and $\geq 3$-year all-cause mortality (multivariate HR 1.37; 95\% CI 1.27-1.48) [16]. Faster and better recovery of left ventricular function [17] and less frequent pulmonary complications [18] after TAVI than after SAVR may also explain the present results. In patients with left ventricular systolic dysfunction, ejection fraction was reported to improve significantly $(p<0.05)$ better at 7 days after TAVI $(32 \pm 9 \%$ to $50 \pm 17 \%)$ than after SAVR $(30 \pm 5 \%$ to $40 \pm 9 \%)$ [17]. The total number of inhospital pulmonary complications per patient was reported to be significantly $(p=0.04)$ lower after TF-TAVI $(1.0 \pm 0.67)$ than after SAVR $(1.8 \pm 0.79)[18]$. However, significantly more frequent major vascular complications (Supplementary Fig. S9) and new permanent 


\title{
Advertisement placed here.
}

\author{
SSS bohn \\ van loghum
}

Houten 2020 


\title{
Advertisement placed here.
}

\author{
SSS bohn \\ van loghum
}

Houten 2020 


\title{
Advertisement placed here.
}

\author{
SSS bohn \\ van loghum
}

Houten 2020 


\title{
Advertisement placed here.
}

\author{
SSS bohn \\ van loghum
}

Houten 2020 
PMI (Supplementary Fig. S10) after TAVI than after SAVR (in the present principal analysis of the secondary end points) might potentially increase allcause mortality. Vascular complications are strongly (approximately 3-fold) associated with increased 30day severe bleeding [19], which may affect 30-day survival after TAVI [15]. Several meta-analyses [20-22], however, indicated that a new permanent PMI was not associated with increased all-cause mortality during follow-up (up to 2 years) after TAVI. Although only the PARTNER 3 trial [6] with low-risk patients indicated a significantly lower incidence of stroke at 1 year (not at 30 days) after TAVI than after SAVR, the other RCTs demonstrated no significant difference in stroke at 30 days and 1 year between TAVI and SAVR, which brought about no significant difference in stroke in the present meta-analysis pooling all the RCTs (Supplementary Fig. S4).

Previous meta-analyses [4, 23-30] indicated no difference in 1-year mortality between TAVI and SAVR. However, these meta-analyses [4, 23-30] (including neither the Evolut Low Risk trial [5] nor the PARTNER 3 trial [6]) were quite different from the present metaanalysis (including both the Evolut Low Risk trial [5] and the PARTNER 3 trial [6]). Longer ( $\geq 2$-year)-term outcomes after TAVI versus SAVR in RCTs have still been inadequate. The longest follow-up durations were 1 year in two RCTs (Evolut Low Risk [5] and PARTNER 3 [6]), 2 years in two RCTs (PARTNER 2 [10] and SURTAVI [11]), and 5 years in three RCTs (NOTION [31], PARTNER 1 [32], and CoreValve U.S. Pivotal High Risk [33]). The present meta-analysis did not analyse $\geq 2$-year mortality after TAVI versus SAVR. There were no statistically significant differences in all-cause mortality between TAVI and SAVR at 2 years $(16.3 \%$ vs $17.9 \%$ [10], $12.6 \%$ vs $14.0 \%$ [11]) and 5 years $(27.7 \%$ vs $27.7 \%$ [31], $74.01 \%$ vs $67.72 \%$ [32], $55.3 \%$ vs $55.4 \%$ [33]) in the as-treated population, despite significantly lower 30-day and 1-year mortality after TAVI than after SAVR being demonstrated in the present study. This 'catch-up' phenomenon at $\geq 2$ years may be owing to more frequent moderate/severe paravalvular aortic regurgitation [4, 34-37] and new-onset left bundle branch block [38], which are associated with higher $\geq 1$-year all-cause [39, 40] and cardiac [41] mortality after TAVI than after SAVR. Although the 5-year results of the Evolut Low Risk, PARTNER 2, PARTNER 3, and SURTAVI trials would be expected in the future, long-term mortality after TAVI might be similar to that after SAVR.

The present study had the following limitations. First, the RCTs included in the present meta-analysis were heterogeneous. Of the seven RCTs, three consisted of patients at low risk (STS-PROM $<4 \%$ ), three were composed of those at intermediate risk (STS-ROM 4-8\%), and only one was made up of those at high risk (STS-ROM $\geq 8 \%$ ). The proportion of patients undergoing TF-TAVI also ranged from 70.1 to $100 \%$ (Tab. 1). The meta-regression analyses, however, demonstrated that mean STS-PROM of patients and proportion of patients undergoing TF-TAVI did not modulate RDs of mortality between TAVI and SAVR. Furthermore, various TAVI valves were used in the RCTs and included CoreValve [5, 8, 11, 12], Evolut R [5, 11], Evolut PRO [5], SAPIEN [9], SAPIEN XT [10], and SAPIEN 3 [6], which may bias the present results. Second, although publication bias favouring TAVI may influence the present results, the funnel plot analysis did not indicate funnel plot asymmetry. Third, the cause of death was not addressed in the present meta-analysis because detailed patient-level data in all RCTs were unavailable. Individual patient data meta-analysis, the gold standard regarding data availability, would be required.

In conclusion, the best evidence from the present meta-analysis of all the currently available RCTs suggests that TAVI may reduce 30-day and 1-year allcause mortality compared with SAVR for AS patients. The present findings must be novel because none of the included RCTs showed significantly lower allcause mortality after TAVI than after SAVR.

Open Access This article is licensed under a Creative Commons Attribution 4.0 International License, which permits use, sharing, adaptation, distribution and reproduction in any medium or format, as long as you give appropriate credit to the original author(s) and the source, provide a link to the Creative Commons licence, and indicate if changes were made. The images or other third party material in this article are included in the article's Creative Commons licence, unless indicated otherwise in a credit line to the material. If material is not included in the article's Creative Commons licence and your intended use is not permitted by statutory regulation or exceeds the permitted use, you will need to obtain permission directly from the copyright holder. To view a copy of this licence, visit http://creativecommons.org/licenses/by/4.0/.

\section{References}

1. Kheiri B, Osman M, Abubakar H, et al. Transcatheter versus surgical aortic valve replacement in low-risk surgical patients: a meta-analysis of randomized clinical trials. Cardiovasc Revasc Med. 2019;20:838-42.

2. Liu Z, Kidney E, Bem D, et al. Transcatheter aortic valve implantation for aortic stenosis in high surgical risk patients: a systematic review and meta-analysis. PLoS ONE. 2018;13:e196877.

3. Wang Y, Zhou Y, Zhang L, Zhu J. Midterm outcome of transcatheter versus surgical aortic valve replacement in low to intermediate risk patients: a meta-analysis of randomized controlled trials. JCardiol. 2018;71:534-9.

4. Burrage M, Moore P, Cole C, et al. Transcatheter aortic valve replacement is associated with comparable clinical outcomes to open aortic valve surgery but with a reduced length of in-patient hospital stay: a systematic review and meta-analysis of randomised trials. Heart Lung Circ. 2017;26:285-95.

5. Popma JJ, Deeb GM, Yakubov SJ, et al. Evolut Low Risk Trial Investigators. Transcatheter aortic-valve replacement with a self-expanding valve in low-risk patients. N Engl J Med. 2019;380:1706-15.

6. PARTNER 3 Investigators, MackMJ, Leon MB, Thourani VH, et al. Transcatheter aortic-valve replacement with a bal- 
loon-expandable valve in low-risk patients. N Engl J Med. 2019;380:1695-705.

7. Nielsen HH, Klaaborg KE, Nissen $\mathrm{H}$, et al. A prospective, randomised trial of transapical transcatheter aortic valve implantation vs. surgical aortic valve replacement in operable elderly patients with aortic stenosis: the STACCATO trial. EuroIntervention. 2012;8:383-9.

8. Thyregod HG, Steinbrüchel DA, Ihlemann N, et al. Transcatheter versus surgical aortic valve replacement in patients with severe aortic valve stenosis: 1-year results from the all-comers NOTION randomized clinical trial. JAm Coll Cardiol. 2015;65:2184-94.

9. PARTNER Trial Investigators, Smith CR, Leon MB, Mack MJ, et al. Transcatheter versus surgical aortic-valve replacement in high-risk patients. N Engl J Med. 2011;364:2187-98.

10. PARTNER 2 Investigators, Leon MB, Smith CR, Mack MJ, et al. Transcatheter or surgical aortic-valve replacement in intermediate-risk patients. NEngl J Med. 2016;374:1609-20.

11. SURTAVI Investigators, Reardon MJ, Van Mieghem NM, Popma JJ, et al. Surgical or transcatheter aortic-valve replacement in intermediate-risk patients. N Engl J Med. 2017;376:1321-31.

12. U.S. CoreValve Clinical Investigators, Adams DH, Popma JJ, Reardon MJ, et al. Transcatheter aortic-valve replacement with a self-expanding prosthesis. N Engl J Med. 2014;370:1790-8.

13. Shrier I, Steele RJ, Verhagen E, Herbert R, Riddell CA, Kaufman JS. Beyond intention to treat: what is the right question? Clin Trials. 2014;11:28-37.

14. Walter SD. Choice of effect measure for epidemiological data. JClin Epidemiol. 2000;53:931-9.

15. Wang J, Yu W, Jin Q, et al. Risk factors for post-TAVI bleeding according to the VARC-2 bleeding definition and effect of the bleeding on short-term mortality: a meta-analysis. Can J Cardiol. 2017;33:525-34.

16. Liao YB, Deng XX, Meng Y, et al. Predictors and outcome of acute kidney injury after transcatheter aortic valve implantation: a systematic review and meta-analysis. EuroIntervention. 2017;12:2067-74.

17. Bauer F, Coutant V, Bernard M, et al. Patients with severe aortic stenosis and reduced ejection fraction: earlier recovery of left ventricular systolic function after transcatheter aortic valve implantation compared with surgical valve replacement. Echocardiography. 2013;30:865-70.

18. PettetJK, McGhee MN, McIlrath ST, Collins GL. Comparison of pulmonary complications in patients undergoing transcatheter aortic valve implantation versus open aortic valve replacement. JCardiothorac Vasc Anesth. 2014;28:497-501.

19. SunY,LiuX, ChenZ, etal. Meta-analysis of predictors of early severe bleeding in patients who underwent transcatheter aortic valve implantation. Am J Cardiol. 2017;120:655-61.

20. Escárcega RO, Magalhaes MA, Lipinski MJ, et al. Mortality in patients requiring pacemaker implantation following transcatheter aortic valve replacement: insights from a systematic reviewand meta-analysis. Int Cardiol. 2014;174:207-8.

21. RegueiroA,Abdul-JawadAltisentO, etal. Impact of new-onset left bundle branch block and periprocedural permanent pacemaker implantation on clinical outcomes in patients undergoing transcatheter aortic valve replacement: a systematic review and meta-analysis. Circ Cardiovasc Interv. 2016;9:e3635.

22. Mohananey D, Jobanputra Y, Kumar A, et al. Clinical and echocardiographic outcomes following permanent pacemaker implantation after transcatheter aortic valve replacement: meta-analysis and meta-regression. Circ Cardiovasc Interv. 2017;10:e5046.
23. Ueshima D, Fovino LN, D'Amico G, Brener SJ, Esposito G, Tarantini G. Transcatheter versus surgical aortic valve replacement in low- and intermediate-risk patients: an updated systematic review and meta-analysis. Cardiovasc IntervTher. 2019;34:216-25.

24. Arora S, Vaidya SR, Strassle PD, et al. Meta-analysis of transfemoral TAVRversus surgical aortic valvereplacement. Catheter Cardiovasc Interv. 2018;91:806-12.

25. Singh K, Carson K, Rashid MK, et al. Transcatheter aortic valve implantation in intermediate surgical risk patients with severe aortic stenosis: a systematic review and metaanalysis. Heart Lung Circ. 2018;27:227-34.

26. Khan SU, Lone AN, Saleem MA, Kaluski E. Transcatheter vs surgical aortic-valve replacement in low- to intermediatesurgical-risk candidates: a meta-analysis and systematic review. Clin Cardiol. 2017;40:974-81.

27. Tam DY, Vo TX, Wijeysundera HC, et al. Transcatheter vs surgical aortic valve replacement for aortic stenosis in lowintermediate risk patients: a meta-analysis. Can J Cardiol. 2017;33:1171-9.

28. Garg A, Rao SV, Visveswaran G, et al. Transcatheter aortic valve replacement versus surgical valvereplacementinlowintermediate surgical risk patients: a systematic review and meta-analysis. J Invasive Cardiol. 2017;29:209-16.

29. Sardar P, Kundu A, Chatterjee S, et al. Transcatheter versus surgical aortic valve replacement in intermediaterisk patients: evidence from a meta-analysis. Catheter Cardiovasc Interv. 2017;90:504-15.

30. Zhou Y, Wang Y, Wu Y, Zhu J. Transcatheter versus surgical aortic valve replacement in low to intermediate risk patients: a meta-analysis of randomized and observational studies. Int JCardiol. 2017;228:723-8.

31. Thyregod HGH, Ihlemann N, Jørgensen TH, et al. Five-year clinical and echocardiographic outcomes from the nordic aortic valve intervention (NOTION) randomized clinical trial in lower surgical risk patients. Circulation. 2019; https://doi.org/10.1161/CIRCULATIONAHA.118.036606.

32. PARTNER 1 trial investigators, MackMJ,Leon MB, Smith CR, et al. 5-year outcomes of transcatheter aortic valve replacement or surgical aortic valve replacement for high surgical risk patients with aortic stenosis (PARTNER 1): a randomised controlled trial. Lancet. 2015;385:2477-84.

33. Gleason TG, Reardon MJ, Popma JJ, et al. CoreValve U.S. pivotal high risk trial clinical investigators. 5-year outcomes of self-expanding transcatheter versus surgical aortic valve replacement in high-risk patients. J Am Coll Cardiol. 2018;72:2687-96.

34. Khan AR, Khan S, Riaz H, et al. Efficacy and safety of transcatheter aortic valve replacement in intermediate surgical risk patients: a systematic review and meta-analysis. Catheter Cardiovasc Interv. 2016;88:934-44.

35. Indraratna P, Tian DH, Yan TD, Doyle MP, Cao C. Transcatheter aortic valve implantation versus surgical aortic valve replacement: a meta-analysis of randomized controlled trials. Int J Cardiol. 2016;224:382-7.

36. Gargiulo G, Sannino A, Capodanno D, et al. Transcatheter aortic valve implantation versus surgical aortic valve replacement: a systematic review and meta-analysis. Ann Intern Med. 2016;165:334-44.

37. Arora S, Strassle PD, Ramm CJ, et al. Transcatheter versus surgical aortic valve replacement in patients with lower surgical risk scores: a systematic review and meta-analysis of early outcomes. Heart Lung Circ. 2017;26:840-5.

38. Massoullié G, Bordachar P, Ellenbogen KA, et al. New-onset left bundle branch block induced by transcutaneous aortic valve implantation. Am J Cardiol. 2016;117:867-73. 
39. Takagi H, Umemoto T, ALICE (All-Literature Investigation of Cardiovascular Evidence) Group. Impact of paravalvular aortic regurgitation after transcatheter aortic valve implantation on survival. Int J Cardiol. 2016;221:46-51.

40. Athappan G, Patvardhan E, Tuzcu EM, et al. Incidence, predictors, and outcomes of aortic regurgitation after transcatheter aortic valve replacement: meta-analysis and systematic review of literature. J Am Coll Cardiol. 2013;61:1585-95.
41. Regueiro A, Abdul-Jawad Altisent O, Del Trigo M, et al. Impact of new-onset left bundle branch block and periprocedural permanent pacemaker implantation on clinical outcomes in patients undergoing transcatheter aortic valve replacement: a systematic review and meta-analysis. Circ Cardiovasc Interv. 2016;9:e3635. 